\title{
Short- and Long-Term Functional Connectivity Differences Associated with Alzheimer's Disease Progression
}

\author{
Jaime D. Mondragón ${ }^{a, b}$ Ramesh Marapin ${ }^{a}$ Peter Paul De Deyn ${ }^{a, b, c}$ \\ Natasha Maurits $^{a, b}$ for the Alzheimer's Disease Neuroimaging Initiative \\ aDepartment of Neurology, University of Groningen, University Medical Center Groningen, Groningen, The \\ Netherlands; b Alzheimer Center Groningen, University of Groningen, University Medical Center Groningen, \\ Groningen, The Netherlands; ' Laboratory of Neurochemistry and Behavior, Institute Born-Bunge, University of \\ Antwerp, Antwerp, Belgium
}

\section{Keywords}

Alzheimer's disease · Alzheimer's Disease Neuroimaging Initiative $\cdot$ Cognitive decline $\cdot$ Progression $\cdot$ Functional connectivity · Mild cognitive impairment

\begin{abstract}
Introduction: Progression of amnestic mild cognitive impairment $(\mathrm{aMCl})$ to Alzheimer's disease $(\mathrm{AD})$ is a clinical event with highly variable progression rates varying from $10-15 \%$ up to $30-34 \%$. Functional connectivity (FC), the temporal similarity between spatially remote neurophysiological events, has previously been reported to differ between $\mathrm{aMCl}$ patients who progress to $\mathrm{AD}(\mathrm{pMCl})$ and those who do not (i.e., remain stable; $\mathrm{SMCl}$ ). However, these reports had a short-term follow-up and do not provide insight into long-term AD progression. Methods: Seventy-nine participants with a baseline and 78 with a 12-month, 51 with a 24-month, and 22 with a +48 -month follow-up resting-state $\mathrm{fMRI}$ with aMCl diagnosis from the Alzheimer's Disease Neuroimaging Initiative database were included. FC was assessed using the CONN toolbox. Local correlation and group
\end{abstract}

independent component analysis were utilized to compare regional functional coupling and between-network FC, respectively, between $\mathrm{sMCl}$ and $\mathrm{pMCl}$ groups. Two-sample $t$ tests were used to test for statistically significant differences between groups, and paired $t$-tests were used to assess cognitive changes over time. Results: All participants (i.e., 66 $\mathrm{sMCl}$ and $19 \mathrm{pMCl}$ ) had a baseline and a year follow-up fMRI scan. Progression from $\mathrm{aMCl}$ to $\mathrm{AD}$ occurred in 19 patients (10 at 12 months, 5 at 24 months, and 4 at $>48$ months), while $73 \mathrm{MCl}$ patients remained cognitively stable $(\mathrm{sMCl})$. The $\mathrm{pMCl}$ and $\mathrm{sMCl}$ cognitive profiles were different. More between-network FC than regional functional coupling differences were present between $\mathrm{sMCl}$ and $\mathrm{pMCl}$ patients. Activation in the salience network (SN) and the default mode network (DMN) was consistently different between sMCl and $\mathrm{pMCl}$ patients across time. Discussion: $\mathrm{sMCl}$ and $\mathrm{pMCl}$ patients have different cognitive and FC profiles. Only pMCl patients showed cognitive differences across time. The DMN and SN showed local correlation and between-network FC differences between the $\mathrm{sMCl}$ and $\mathrm{pMCl}$ patient groups at multiple moments in time.

(c) 2021 The Author(s).

Published by S. Karger AG, Basel karger@karger.com www.karger.com/dee

Karger $\stackrel{\text { ' }}{5}$

BOPEN ACCESS
(C) 2021 The Author(s)

Published by S. Karger AG, Basel

This article is licensed under the Creative Commons Attribution 4.0 International License (CC BY) (http://www.karger.com/Services/ OpenAccessLicense). Usage, derivative works and distribution are permitted provided that proper credit is given to the author and the original publisher.
Correspondence to:

Jaime D. Mondragón, j.d.mondragon.uribe@ umcg.nl 


\section{Introduction}

The National Institute on Aging at National Institutes of Health and the Alzheimer's Association (NIA-AA) Preclinical Workgroup proposes a theoretical continuum of Alzheimer's disease (AD), which includes 3 conditions, a preclinical, a prodromal, and a clinical stage, which comprise mild, moderate, and severe dementia. Mild cognitive impairment (MCI), the transitional cognitive state between normal aging and mild dementia [1], is considered a prodromal stage of $\mathrm{AD}$ [2]. Due to its emphasis on memory loss, amnestic mild cognitive impairment (aMCI) has been associated with an increased risk of developing $\mathrm{AD}$. Progression of aMCI to $\mathrm{AD}$ (pMCI) is a clinical event; however, progression rates are highly variable. Some studies report annual progression rates of 10 $15 \%$ [1], while others report progression rates of $30-34 \%$ [3-5]. These wide ranges can be partially explained by classification, recruitment, and demographic factors. However, this variability may be further understood by investigating how brain functioning differs between pMCI and aMCI patients who do not progress, so-called stable MCI (sMCI) patients. Changes in functional connectivity (FC) assessed using resting-state fMRI (rsfMRI) that is assumed to reflect functional integration between different brain regions could assist in providing a comprehensive biomarker profile of the aging brain.

FC can be defined as the temporal similarity between spatially remote neurophysiological events [6]. FC analytic approaches can be divided into functional segregation measures (e.g., amplitude of low-frequency fluctuations, regional homogeneity, integrated local correlation, and local efficiency) and functional integration approaches (e.g., seed-based analysis, independent component analysis [ICA], and graph analysis) [7]. While functional segregation analytic approaches provide insight into the local function of specific brain regions, functional integration analytic approaches focus on the functional relationship between different brain areas. A complete picture of brain network functioning can thus be obtained by employing a functional segregation approach, as well as a functional integration analytic one, thereby analyzing "within-network" and "between-network" FC, respectively. Here we use local correlation analysis as a measure of functional segregation, which focuses on the local function of specific brain regions by assessing regional functional coupling [8]. As a voxel-to-voxel measure of functional integration, we use ICA, which is straightforward to calculate and applicable to functional networks [9].
With age, brain functional segregation significantly decreases $[10,11]$. These changes are expressed in decreased functional segregation metrics such as modularity and local efficiency, especially in networks supporting higher level cognitive functions like the default mode network (DMN), the cingulo-opercular network, and frontoparietal control network [12]. Compared to aMCI patients, healthy older adults preserve more within-network connections (i.e., functional segregation) and have also more between-network and cross-hemispheric connections (i.e., functional integration) [13]. Network connectivity has the potential to become an $\mathrm{AD}$ continuum biomarker, as functional network reorganization is found in aMCI patients [14-16]. Short-term follow-up (i.e., 1 year) might, however, be too short to assess differences in FC between pMCI and sMCI, as conflicting results have been reported regarding the global network organization (i.e., using graph theoretical analysis) of pMCI compared to sMCI. One study found a decrease in global FC (i.e., decreased functional integration such as expressed in lower mean clustering) and decreased regional FC (as expressed in a decreased path length) in pMCI compared to sMCI [17], while another study found no differences between the 2 groups in whole-brain functional network organization (i.e., functional integration) after 1 year [18]. However, both studies of whole-brain and regional FC comparing $\mathrm{pMCI}$ and sMCI patients have found decreased FC over time in pMCI. Three cross-sectional studies that assessed aMCI patients over a 2-year and one over a 3-year window (i.e., 2 and 3 cross-sectional time-points) reported more disruptions and connectivity changes in pMCI than in sMCI compared to healthy control (HC) participants. When compared to HC, more whole-brain network disruptions (i.e., functional integration) and loss of connections between neighboring areas (i.e., functional segregation) were observed in pMCI than when sMCI patients were compared to healthy subjects $[17,19]$. Reduced FC between the entorhinal and hippocampal regions (i.e., functional integration) has been reported in pMCI and not in sMCI when being compared to HC [20]; in addition, a gradual and progressive decrease in regional FC (i.e., functional integration; between left angular gyrus and bilateral inferior parietal lobules, between dorsolateral prefrontal and lateral temporal cortices, and between the left middle occipital gyrus and middle occipital gyri) has been observed in pMCI compared to sMCI [21]. FC has provided insight into the phenomenon of $\mathrm{AD}$ progression; however, understanding of how FC changes over time in this patient group is still scarce. 
To further explore the differences in brain FC among aMCI patients and how these differences are associated with progression from aMCI to $\mathrm{AD}$, we identified several networks of highly connected areas from the Alzheimer's Disease Neuroimaging Initiative (ADNI) cohort using a data-driven approach. This work aimed to assess the cognitive differences between- and within-groups and the cross-sectional group differences in FC at baseline, and follow-up at 12 months, 24 months, and $>48$ months (i.e., long term) between pMCI and sMCI patients. The primary aim of this study was to assess brain FC of aMCI patients, specifically how FC differs between sMCI and pMCI patients across 4 time-points.

\section{Methods}

\section{Description of ADNI Data Set}

Data were obtained from the ADNI database (http://adni.loni. usc.edu/data-samples/access-data/). ADNI is a multicenter collaboration launched in 2004, with the common goal of collecting, validating, and utilizing data such as magnetic resonance imaging (MRI) and positron emission tomography images, genetics, cognitive tests, cerebrospinal fluid (CSF), and blood biomarkers as biomarkers to define AD progression [22]. Participants included in the ADNI project are between the ages of 55 and 90 years, completed at least 6 years of education, and are free of any significant neurological disease other than $\mathrm{AD}$. With the goal of developing new treatments and the optimization of clinical trials for MCI and AD populations, ADNI was initiated to define clinical changes (e.g., related to clinical diagnosis and neuropsychological assessment) and biomarker profiles (e.g., imaging and CSF) [23]. The entire data set was downloaded from the ADNI-1, ADNI-2, and ADNI Grand Opportunity (ADNI-GO) databases beginning on August 28, 2018, and ending on February 15, 2019. Participant eligibility criteria for ANDI-1, ADNI-2, and ADNI-GO are identi$\mathrm{cal}$ and can be found in the ADNI General Procedures Manual (ADNI-I, http://adni.loni.usc.edu/methods/documents/). The ADNI has developed harmonized standard operating procedures for sample collection, processing, and handling of CSF and serum biomarkers [23]. The ADNI was approved by the Institutional Review Boards of all the participating centers. Written informed consent was obtained from all patients. For more information, we refer the reader to www.adni-info.org.

\section{Description of Participants}

For our current study, we included participants from the ADNI-1, ADNI-2, and ADNI-GO databases who had at least a baseline and a 1-year follow-up rs-fMRI scan. One-year follow-up was defined as rs-fMRI acquisitions within 10 and 14 months from baseline image acquisition. The diagnostic inclusion criteria were based on the ADNI protocols available on the ADNI website. Clinical diagnosis at each time-point was based on the multidisciplinary consensus diagnosis from each ADNI site, which ranged between cognitively normal, $\mathrm{aMCI}$, and $\mathrm{AD}$; for progression classification purposes, those selection criteria are presented in the next section. Briefly, clinical diagnosis was assigned to the partici- pants by the site investigators and reassessed at each visit. For this study, we used the diagnosis assigned during the first scan and not the diagnosis the patient had upon enrollment to the ADNI project. MCI patients had Mini-Mental State Examination (MMSE) scores $\geq 24$, a global clinical dementia rating (CDR) score of 0.5 , objective memory loss measured by education adjusted scores on the Wechsler Memory Scale Logical Memory II, absence of significant levels of impairment in other cognitive domains, preserved activities of daily living, and absence of dementia. Demographical, neuropsychological, biomarker, and neuroimaging data were extracted from the 3 previously mentioned ADNI data sets (online suppl. Table 1; for all online suppl. material, see www.karger.com/doi/10.1159/000518233). Exclusion criteria were defined by the ADNI study protocol [24]. Functional MRI, fluid-attenuated inverse recovery images, and volumetric $\mathrm{T} 1$-weighted images were downloaded for all participants with a baseline and a 1-year follow-up assessment, as well as these MRI sequences for those participants with available follow-up scan sessions at 24 months ( \pm 2 months) and at $>48$ months from the initial fMRI scan session. Other neuropsychological, biomarker, and neuroimaging data for the 24-month follow-up visit and for the $\geq 48$-month follow-up visit were also extracted from the ADNI-1, ADNI-2, and ADNIGO databases for all participants included for whom that information was available. The Hachinski ischemic score was calculated for every participant at each visit based on the clinical characteristics and accompanying signs and symptoms (e.g., dementia onset, clinical evolution, confusion, personality and emotional changes, depression, somatic complaints, history of hypertension and strokes, and focal neurological signs and symptoms) from the ADNI clinical data. All patients included had a Hachinski score $\leq 4$. Visual inspection for hyperintensities in the fluid-attenuated inverse recovery scan to detect possible ischemic lesions was performed by one of the authors (J.D.M.) and corroborated through the "MRI_Infarct" data set to exclude participants with large vascular lesions.

\section{Assessment of AD Progression and Reversion}

Progression from MCI to $\mathrm{AD}$ was assessed on each visit and was based on the progression of a global CDR score from 0.5 to $\geq 1.0$ and fulfillment of the NINCDS-ADRDA criteria for probable $\mathrm{AD}$ or later adopted NIA-AA revised criteria for probable $\mathrm{AD}$ (pMCI) [25]. Patients with MCI who improved on their neuropsychological and clinical assessment and displayed normal cognition in subsequent clinical visits were designated as patients who underwent cognitive "reversion" (rMCI). The clinical diagnosis and progression status were assigned to the participants by the site investigators and reassessed at each visit; meanwhile, the cognitive reversion was interpreted by the authors from the clinical diagnoses provided for each patient's visits.

\section{Cognitive Assessment}

Cognitive data were extracted from the "ADNIMERGE" file, which incorporates merged data sets containing data from ADNI $1 / \mathrm{GO} / 2$ clinical data and numeric summaries to assess cognitive function over time and to perform between-group comparisons. The neuropsychological variables used in the analysis of cognitive changes were the CDR sum of boxes (CDR-SOB), MMSE, Montreal Cognitive Assessment (MoCA), and the 11-item Alzheimer's Disease Assessment Scale-Cognitive Subscale (ADAS-Cog). The global CDR score was calculated from the CDR-SOB, where a 
CDR-SOB between 0.5 and 4.0 corresponded to a global CDR score of 0.5 , a CDR-SOB between 4.5 and 9.0 corresponded to a global CDR score of 1.0, a CDR-SOB between 9.5 and 15.5 corresponded to a global CDR score of 2.0, and a CDR-SOB between 16.0 and 18.0 corresponded to a global CDR score of 3.0 [26]. CDR was used to assess disease progression, $\mathrm{AD}$ progression, and reversion from MCI to normal cognition. Global cognition was assessed through MMSE scores, MoCA scores, and the 11-item ADAS-Cog scores.

\section{MRI Image Acquisition}

All MRI scans were performed on Philips 3T MRI scanners, using an 8-channel head matrix coil. High-resolution volumetric T1weighted imaging was acquired using a 3D MP-RAGE sequence, with whole-brain coverage and $1 \times 1 \times 1.2 \mathrm{~mm}$ voxel resolution. The rs-fMRI images were acquired using a single-shot T2*-weighted EPI sequence collecting 140 volumes, using a TR of 3,000 ms, a flip angle of $80^{\circ}$, and $3.3 \mathrm{~mm}$ isotropic resolution. The participants kept their eyes open fixated on a point displayed on a screen for all rs-fMRI scans. Full descriptions of ADNI MRI image acquisition protocols are available at http://adni.loni.usc.edu/methods/documents/mri-protocols/.

\section{fMRI Image Preprocessing}

The fMRI image preprocessing was performed using the SPM 12 software package (Wellcome Trust Centre for Neuroimaging, University College London, United Kingdom, http://www.fil.ion. ucl.ac.uk/spm/software) implemented in MatLab 2018b (MathWorks, Natick, MA). All preprocessing steps were performed using the CONN toolbox (Functional Connectivity SPM Toolbox 2017, McGovern Institute for Brain Research, Massachusetts Institute of Technology, http://ww.nitrc.org/projects/conn) following the default preprocessing pipeline for volume-based analyses [27]. The preprocessing included the following steps: (1) realignment and unwarping; (2) slice-timing correction; (3) structural segmentation and normalization; (4) functional normalization; (5) outlier identification; and (6) functional smoothing.

In brief, the first 10 volumes were discarded to allow for equilibration of the magnetic field. All remaining volumes were realigned with the first volume to correct for motion. The realigned images were slice-time corrected, followed by tissue segmentation (i.e., gray matter/white matter/CSF normalized masks were determined) and coregistration to a T1-weighted Montreal Neurological Institute native space. Normalization was performed using DARTEL [28] with isotropic $2 \mathrm{~mm}$ voxels. Outlier identification was performed using Artifact Detection Tools, which compute regressors for outliers and movement (i.e., resulting in scrubbing parameters). Spatial smoothing was performed using an $8-\mathrm{mm}$ full-width at half-maximum Gaussian kernel. Subject movement realignment and scrubbing parameters (using conservative settings for functional outlier detection settings, global signal $z$-value threshold, and subject motion of $0.5 \mathrm{~mm}$ ) were assigned as firstlevel covariates. Quality assurance (QA) plots were visually inspected to detect other possible outliers (i.e., "QA_ValidScans," "QA_MaxMotion," and "QA_InvalidScans") and inspected for an adequate match with Montreal Neurological Institute space and proper coregistration across participants. Preprocessing using the CONN default preprocessing pipeline thus yielded normalized structural volumes, gray matter/white matter/CSF normalized masks, realigned slice-time corrected, and normalized smoothed functional volumes, as well as subject-level movement and scrubbing related first-level covariates. After the anatomical and functional preprocessing steps, a denoising step was included to define, explore, and remove possible confounds in the BOLD signal. The denoising step applies linear regression and band-pass (i.e., 0.01$0.1 \mathrm{~Hz}$ ) filtering to remove unwanted motion, white matter, and CSF noise components, as well as physiological noise sources, hence reducing spurious sources of variance in fMRI.

\section{fMRI Processing and Connectivity Analysis}

Following preprocessing, rs-fMRI data were processed using the CONN toolbox. Local correlation analysis was used as a voxelto-voxel measure of functional segregation for each observational point. Local correlation is a measure of local functional coupling for each voxel that is determined by the average correlation between the time courses in each seed voxel and its neighbors. A neighborhood of a voxel is defined as the probabilistic region delimited by an isotropic Gaussian kernel. In this study, we used an 8-mm kernel, which is conventionally used by the authors of the CONN toolbox [27]. To determine between-network FC, we used another voxel-to-voxel approach, a group-ICA to identify functional brain networks. ICA as applied to functional MRI is a datadriven method that attempts to separate independent sources either spatially or temporally by organizing brain regions with a similar time course of activation into spatially independent patterns of BOLD signal that are represented as independent components [29]. The CONN toolbox incorporates an atlas that includes several commonly used functional brain networks (i.e., default mode, sensorimotor, visual, salience, dorsal attention, frontoparietal, language, and cerebellar) and areas (e.g., medial prefrontal cortex, posterior cingulate cortex, and left and right lateral parietal cortices). The CONN toolbox follows the general methodology described by Calhoun et al. [29], which uses a temporal concatenation of BOLD signal data across multiple participants followed by a group-level dimensionality reduction using principal component analysis, and fast-ICA for estimation of spatially independent components. Furthermore, back-projection for individual subjectlevel spatial map estimation is attained by performing dual regression with a univariate spatial-regression step and a multivariate temporal-regression step [29]. Twenty independent components were chosen as recommended by the CONN toolbox developers, as it allows for adequate characterization and separation of the represented components by matching the independent component to a network template via an automated spatial correlation [27]. To this end, a post hoc Z-statistic was derived from the voxelto-voxel one-sample $t$-tests of each subject-level ICA spatial map with suprathreshold areas to help quantify the spatial overlap between ICs and the network template. This statistic, known as the Dice similarity coefficient or the Sørensen-Dice index, allowed to assign each independent component to a single network. The thresholds selected were 3.5 for the baseline and 12-month analyses, 3.7 for 24 months, and 3.2 for $>48$ months; the threshold for each observational point was adjusted so that there was a one-toone correspondence between components and networks visualized in the spatial correlation maps (i.e., one independent component was equal to a single network).

\section{Statistical Analysis}

Statistical analysis was performed using SPSS 25 (SPSS Inc., Chicago, IL, USA). Data were screened for outliers and normality 


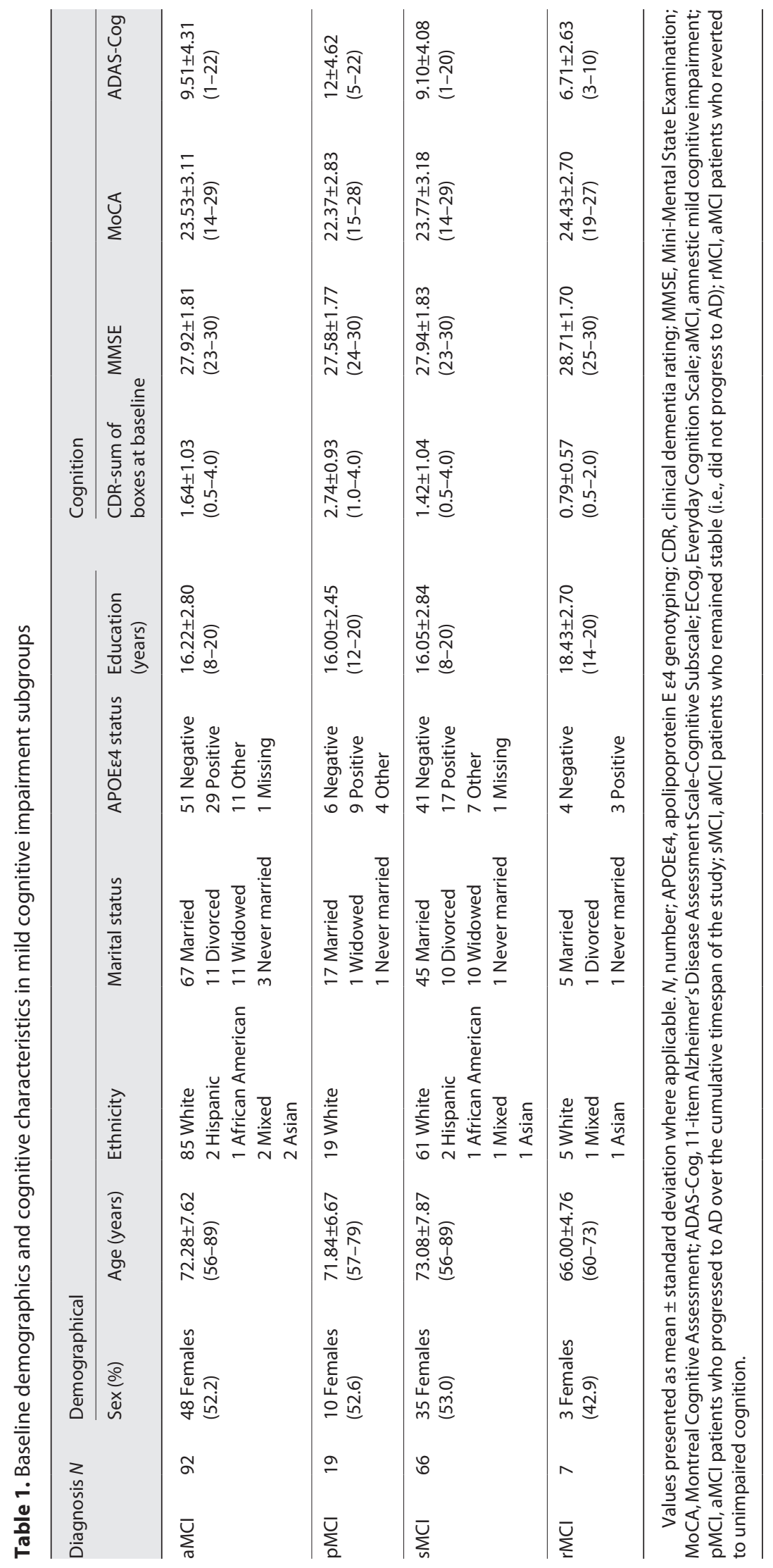




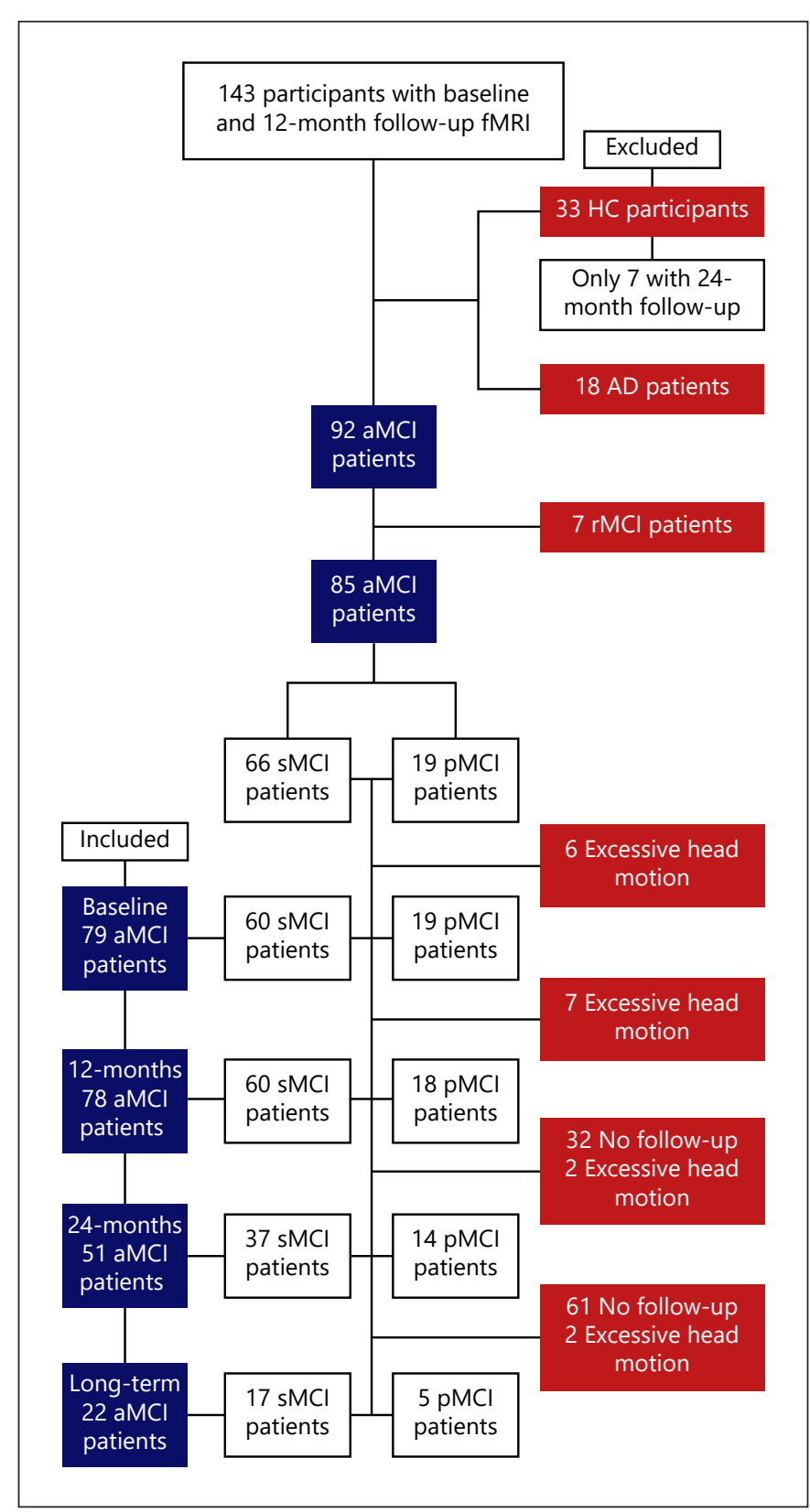

Fig. 1. Participant selection flow diagram. Participant selection at baseline, 12,24 , and $\geq 48$ months. fMRI, functional magnetic resonance; $\mathrm{HC}$, healthy control; $\mathrm{AD}$, Alzheimer's disease clinical diagnosis; aMCI, amnestic mild cognitive impairment; rMCI, aMCI patients who reverted to unimpaired cognition; sMCI, aMCI patients who remained stable (i.e., did not progress to AD); pMCI, aMCI patients who progressed to $\mathrm{AD}$ over the cumulative timespan of the study. aMCI patients are classified as pMCI or sMCI if by the $\geq 48$-month clinical evaluation the patients had progressed or remained stable without reverting to unimpaired cognition (i.e., cumulative progression without reversion over +48 months). assumptions. The normality of continuous variables was assessed with the Shapiro-Wilk normality test and visually using histograms and Q-Q plots. For variables with nonnormal distribution, a Wilcoxon rank-sum test was used. For cognitive function analysis, independent sample $t$-tests were performed to assess the between-group differences (i.e., sMCI vs. pMCI); meanwhile, paired sample $t$-tests were performed to assess the within-group differences between the baseline cognitive level and the 12-, 24-, and +48 -month evaluation points. For the cognitive function longitudinal assessment, paired sample $t$-tests were performed rather than repeated measures ANOVAs for 2 main reasons: first, the number of patients decreased as time progressed, and second, this study aimed to assess the change in global cognitive function compared to the baseline cognitive function rather than global cognition change across time. For voxel-level measurements, two-sample paired $t$-tests were performed on mean regional activation maps to assess between-group differences for the group-ICA and the local correlation analysis; the statistical significance was set at $p \leq 0.05$ FDR-corrected for voxel level and cluster size.

\section{Results}

\section{Participant Characteristics}

Ninety-two participants (i.e., $66 \mathrm{sMCI}, 19 \mathrm{pMCI}$, and 7 aMCI patients who reverted to normal cognition rMCI) had baseline and 12-months' follow-up structural MRI and fMRI data, fulfilling the initial criteria for inclusion in this study. The full description of demographic, diagnostic, and cognitive participant characteristics at baseline can be found in Table 1. Six participants were removed from the baseline sample due to excessive head movement leaving 79 participants (i.e., $60 \mathrm{sMCI}$ and 19 pMCI) with valid scans, while 7 participants were removed due to head movement from the 12-month follow-up leaving 78 subjects (i.e., $60 \mathrm{sMCI}$ and $18 \mathrm{pMCI}$ ). Two participants were removed from the 24-month follow-up sample, leaving 51 participants (i.e., $37 \mathrm{sMCI}$ and 14 pMCI) who were included in the FC analysis; meanwhile, 2 participants were removed from the long-term follow-up sample due to excessive head movement, leaving 22 participants (i.e., $17 \mathrm{sMCI}$ and $5 \mathrm{pMCI}$ ). A participant selection flow diagram is displayed in Figure 1. Sex, age, ethnicity, marital status, APOE\&4 status, and education were assessed for between-group statistical differences, and none were found.

\section{$A D$ Progression and MCI Reversion}

Overall, progression from aMCI to $\mathrm{AD}$ dementia stage (i.e., cumulative progression after 12 months, 24 months, and after $>48$ months; pMCI) occurred in 19 patients (10 at 12 months, 5 at 24 months, and 4 at $>48$ months), while $73 \mathrm{MCI}$ patients remained cognitively stable (sMCI) until 
the end of registration for the ADNI-2 and ADNI-GO phases. Cognitive reversion, defined as aMCI patients who reverted to normal cognition (rMCI), occurred in 7 aMCI patients (5 at 12 months, 1 at 24 months, and 1 at $>48$ months). For a full description of demographic and cognitive data of aMCI and its subgroups at baseline, we refer the reader to Table 1 .

\section{Cognitive Function Between- and Within-Group Differences}

Between-group cognitive function was assessed with CDR-SB, MMSE, and MoCA at all 4 observation points, while ADAS-Cog 11 item was only assessed at baseline and after a year. The mean CDR-SB score was higher for pMCI patients than for sMCI patients at all 4 time-points (i.e., baseline, mean $=2.74 \pm 0.93, T \leq 0.001,95 \mathrm{CI}[0.83$, $1.81] ; 12$ months, mean $=3.92 \pm 1.56, T \leq 0.001,95 \mathrm{CI}$ $[1.95,3.14] ; 24$ months, mean $=4.79 \pm 2.23, T \leq 0.001,95$ CI $[2.67,4.42]$; and +48 months, mean $=6.29 \pm 2.91, T \leq$ $0.001,95$ CI $[3.08,6.49])$. The mean MMSE score was lower in the pMCI than in the sMCI group at 12,24 , and +48 months, but not at baseline (i.e., baseline, mean $=$ $27.58 \pm 1.77, T=0.449,95$ CI $[-0.58,1.30] ; 12$ months, mean $=25.79 \pm 1.99, T \leq 0.001,95 \mathrm{CI}[-3.34,-1.20] ; 24$ months, mean $=24.59 \pm 3.45, T=0.004,95$ CI $[-4.93$, $-1.03]$; and +48 months, mean $=22.57 \pm 4.12, T=0.049$, 95 CI $[-7.84,-0.02])$. Similar to the MMSE, the mean MoCA score was lower at 12,24 , and +48 months, but not at baseline (i.e., baseline, mean $=22.37 \pm 2.83, T=0.075$, 95 CI $[-2.95,0.15] ; 12$ months, mean $=21.53 \pm 2.44, T=$ $0.001,95$ CI $[-3.82,-0.98] ; 24$ months, mean $=19.35 \pm$ $4.32, T=0.001,95 \mathrm{CI}[-6.89,-2.03]$; and +48 months, mean $=15.00 \pm 4.87, T=0.006,95 \mathrm{CI}[-11.96,-2.66])$. Finally, the mean ADAS-Cog score was higher in the pMCI than in the sMCI group at both baseline and the 12 -month follow-up (i.e., baseline, mean $=12.00 \pm 4.62$, $T=0.020,95$ CI $[0.50,5.31]$; and 12 months, mean $=15.40$ $\pm 4.79, T=0.001,95 \mathrm{CI}[2.07,7.93])$. Online suppl. Figure 1 displays the cognitive trajectories of sMCI, pMCI, and rMCI patients.

To understand if the cognitive function varied over time within each of the pMCI and sMCI groups, the difference between the baseline cognition and the three-follow-up time-points was compared. Among sMCI patients, the mean CDR-SB scores were not statistically different between baseline and the other 3 time-points (i.e., 12 months, mean difference $=-0.04 \pm 0.65, T=0.636,95$ CI $[-0.20,0.12] ; 24$ months, mean difference $=0.14 \pm$ $0.83, T=0.921,95$ CI $[-0.27,0.30]$; and +48 months, mean difference $=0.44 \pm 1.09, T=0.130,95 \mathrm{CI}[-0.15$,

Functional Connectivity and AD

Progression
1.02]). The mean MMSE score differences were lower only at +48 months than the baseline score for sMCI patients (i.e., 12 months, mean difference $=0.12 \pm 1.84, T=$ $0.593,95$ CI $[-0.33,0.57] ; 24$ months, mean difference $=$ $-0.43 \pm 2.58, T=0.314,95$ CI $[-1.29,0.43]$; and +48 months, mean difference $=-1.50 \pm 2.19, T=0.015,95 \mathrm{CI}$ $[-2.67,-0.33])$. The mean MoCA group scores were not statistically different between the baseline and remaining follow-up evaluations among sMCI patients (i.e., 12 months, mean difference $=0.63 \pm 2.28, T=0.827,95 \mathrm{CI}$ $[-0.51,0.63] ; 24$ months, mean difference $=0.24 \pm 2.19$, $T=0.504,95$ CI $[-0.49,0.97]$; and +48 months, mean difference $=-0.75 \pm 2.49, T=0.247,95 \mathrm{CI}[-2.08,0.58])$. The mean ADAS-Cog score difference between the baseline and the 12-month follow-up was not statistically significant for the sMCI patient group (i.e., mean difference $=$ $1.31 \pm 6.66, T=0.115,95 \mathrm{CI}[-0.33,2.94])$.

The within-group cognitive function differences between the baseline and the three follow-up time-points were evident in the pMCI patient group. The mean CDRSB group scores were higher at all three follow-up timepoints than the baseline evaluation for $\mathrm{pMCI}$ patients (i.e., 12 months, mean difference $=1.18 \pm 1.34, T=0.001$, 95 CI $[0.54,1.83]$; 24 months, mean difference $=2.09 \pm$ $2.16, T=0.001,95 \mathrm{CI}[0.98,3.20]$; and +48 months, mean difference $=3.64 \pm 2.90, T=0.016,95 \mathrm{CI}[0.96,6.32])$. The mean MMSE group differences were lower at all followup time-points than the baseline evaluation for the pMCI group (i.e., 12 months, mean difference $=-1.79 \pm 1.84, T$ $=0.001,95 \mathrm{CI}[-2.68,-0.90] ; 24$ months, mean difference $=-3.00 \pm 3.46, T=0.003,95$ CI $[-4.20,-1.22]$; and +48 months, mean difference $=5.29 \pm 4.15, T=0.015,95 \mathrm{CI}$ $[-9.13,-1.45])$. The mean MoCA group differences were lower at the 24- and +48-month follow-up time-points for pMCI patients (i.e., 12 months, mean difference $=$ $-0.84 \pm 2.67, T=0.186,95$ CI $[-2.13,0.45] ; 24$ months, mean difference $=-3.18 \pm 3.34, T=0.001,95$ CI $[-4.89$, $-1.46]$; and +48 months, mean difference $=-7.14 \pm 6.39$, $T=0.025,95$ CI $[-13.05,-1.24])$. Lastly, the mean ADASCog group differences between the baseline and the 12-month evaluation for pMCI patients were higher at the follow-up for the pMCI patient group (i.e., mean difference $=3.40 \pm 4.03, T=0.002,95 \mathrm{CI}[1.46,5.34])$.

\section{Group-ICA}

Twenty independent components were chosen (online suppl. Fig. 2-5). A large spatial correlation corresponds to a better match to the network template. After matching, the following components were identified at baseline: components 1 and 12 corresponded to the cerebellar net- 
Fig. 2. FC analysis differences at baseline. Activation maps are graphical representations of online suppl. Table $2 a$, b, where hot colors represent greater mean regional activation (i.e., between clusters for the local correlation analysis and between specific region and independent component for between-network FC analysis) in sMCI than in pMCI patients and cold colors represent lower activation group differences (i.e., cold colors reflect the opposite effect, greater mean regional activation in pMCI than in sMCI patients). Subcortical activation is represented in red. Activation values based on $T$ values (i.e., activation color bar range -5 to 5$)$. FC, functional connectivity.

\section{Local correlation differences at baseline}

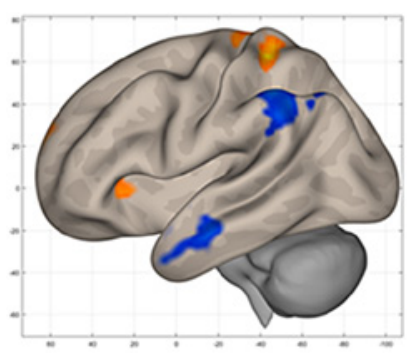

Left view

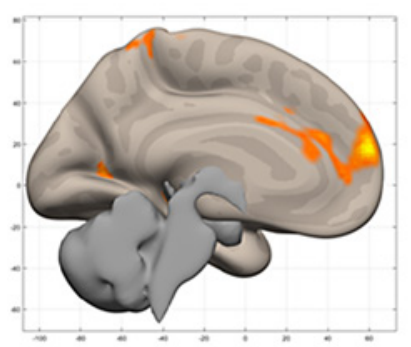

Left medial view

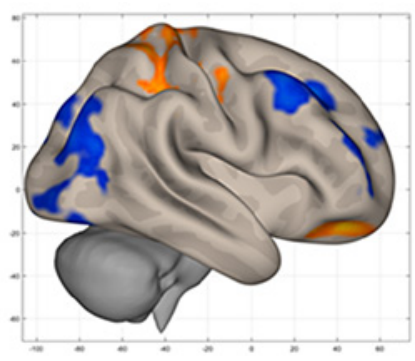

Right view

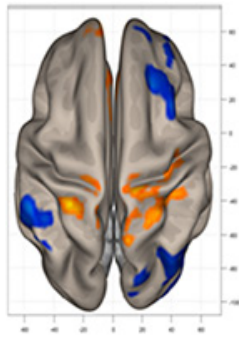

Superior view

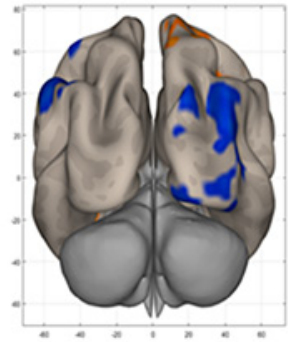

Posterior view

Between-network functional connectivity differences at baseline

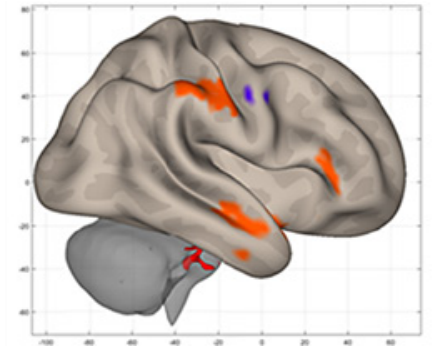

Right view

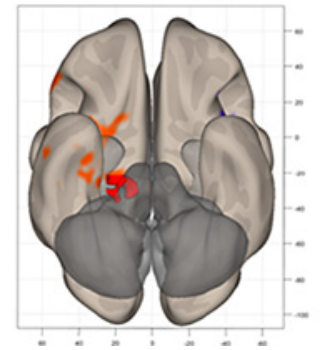

Inferior view

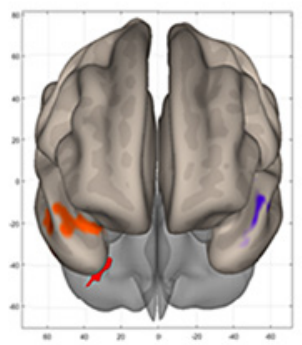

Anterior view
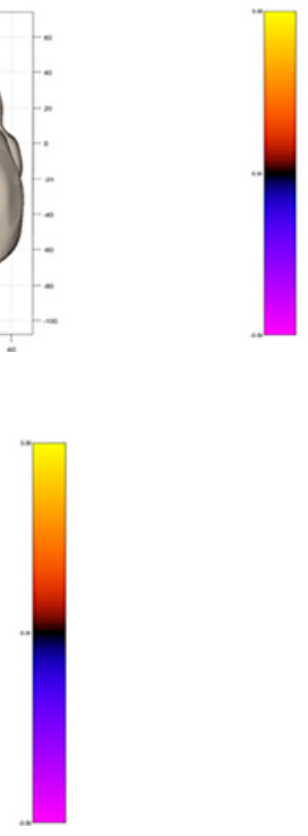

work; components $2,3,7$, and 11 to the DMN; components 4,6 , and 17 to the visual network; component 8 to the salience network (SN); components 9 and 18 to the sensorimotor network; component 10 to the frontoparietal network; component 16 to the dorsal attention net- work; and component 20 to CSF. Online suppl. Figures 2-5 display the spatial correlation of independent components to the template at baseline, at 12 months, at 24 months, and at $\geq 48$ months, respectively. 
Fig. 3. FC analysis differences at baseline + 12 months. Activation maps are graphical representations of online suppl. Table $3 \mathrm{a}, \mathrm{b}$, where hot colors represent greater mean regional activation (i.e., between clusters for the local correlation analysis and between specific region and independent component for between-network FC analysis) in sMCI than in pMCI patients and cold colors represent lower activation group differences (i.e., cold colors reflect the opposite effect, greater mean regional activation in pMCI than in sMCI patients). Subcortical activation is represented in red. Activation values based on $T$ values (i.e., activation color bar range -5 to 5 ). FC, functional connectivity.

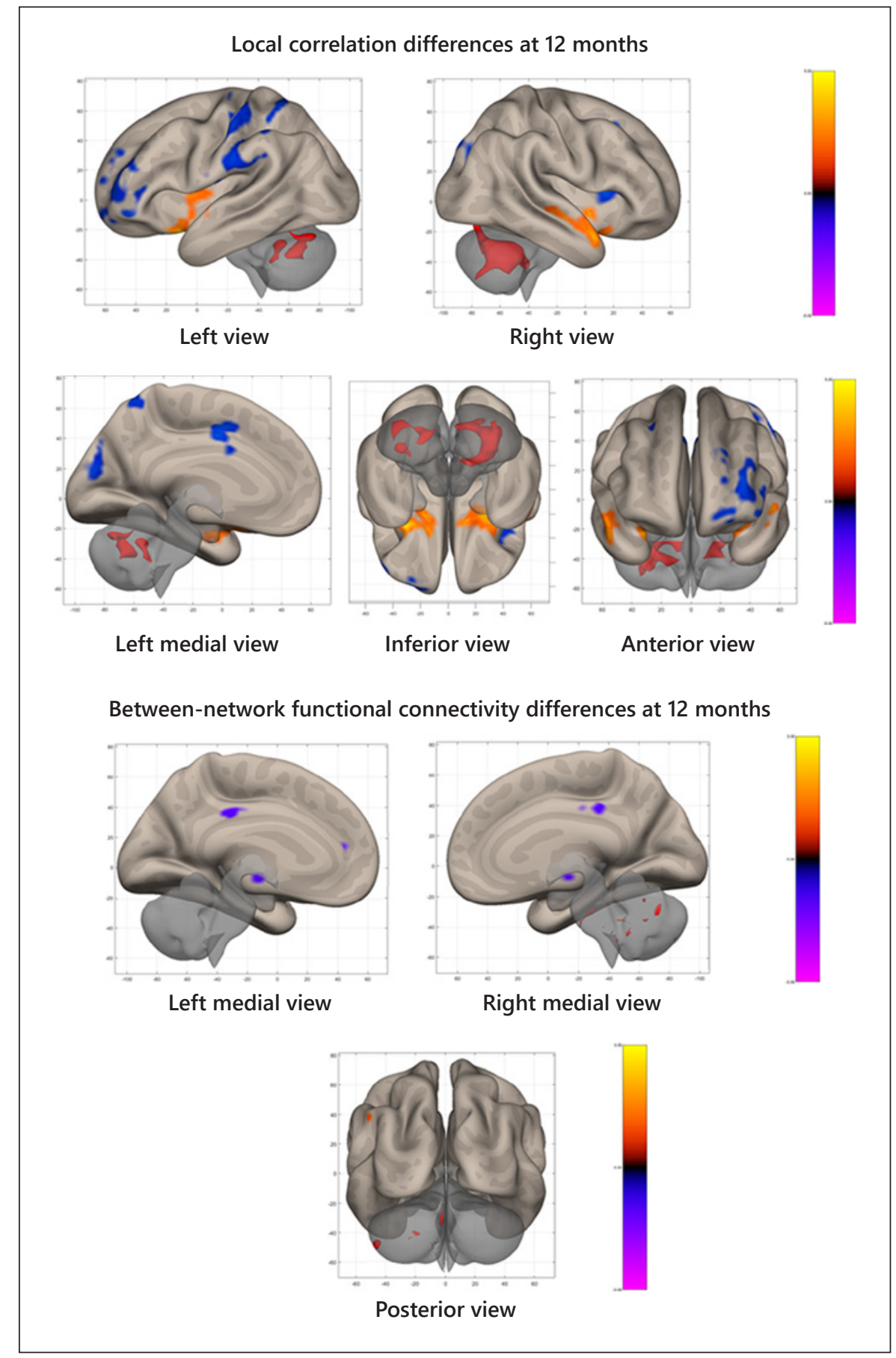

\section{Group-ICA Between-Group Analysis: sMCI and} pMCI

An overview of the local correlation analysis, at baseline, 12 months, 24 months, and $>48$ months, is presented in online suppl. Tables $2 \mathrm{a}-5 \mathrm{a}$. Online suppl. Tables $2 \mathrm{~b}-5 \mathrm{~b}$ provide an overview of the between-network FC at the 4 observational points. Visual representations of brain surface activation maps are depicted in Figures 2-5 for the between-group comparisons between sMCI and pMCI at baseline and 12-, 24-, and >48-month follow-ups, respectively. 
Fig. 4. FC analysis differences at baseline + 24 months. Activation maps are graphical representations of online suppl. Table $4 a, b$, where hot colors represent greater mean regional activation (i.e., between clusters for the local correlation analysis and between specific region and independent component for between-network FC analysis) in sMCI than in pMCI patients and cold colors represent lower activation group differences (i.e., cold colors reflect the opposite effect, greater mean regional activation in pMCI than in sMCI patients). Subcortical activation is represented in red. Activation values based on $T$ values (i.e., activation color bar range -5 to 5 ). FC, functional connectivity.

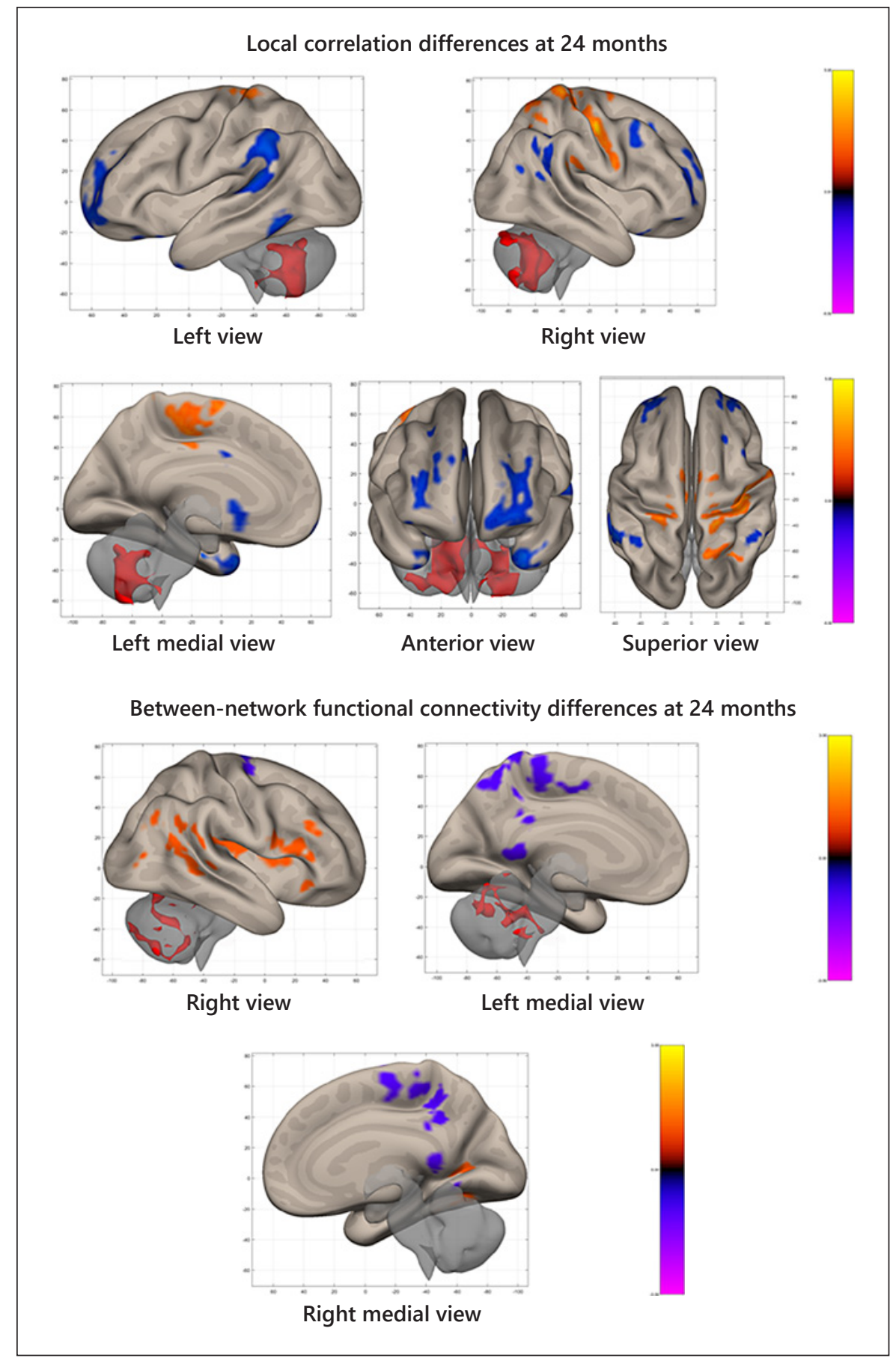

Local Correlation Analysis

At baseline, sMCI participants had greater local correlation than $\mathrm{pMCI}$ patients in the sensorimotor network $(T=5.16, \mathrm{p}-\mathrm{FDR} \leq 0.001)$, the left lateral SN $(T=4.95$, pFDR $\leq 0.001)$, and the DMN $(T=4.35, \mathrm{p}-\mathrm{FDR} \leq 0.001 ; T$ $=3.75, \mathrm{p}$-FDR $\leq 0.001$; here, each $T$-value represents a separate cluster within a particular network). Conversely, at baseline pMCI patients had greater local correlation than sMCI patients in the visual network $(T=6.87$, p$\mathrm{FDR} \leq 0.001)$, the right medial portion of the $\mathrm{SN}(T=5.58$, $\mathrm{p}-\mathrm{FDR} \leq 0.001)$, the DMN $(T=5.45, \mathrm{p}-\mathrm{FDR} \leq 0.001)$, and the language network $(T=4.86, \mathrm{p}-\mathrm{FDR} \leq 0.001$; online suppl. Table 2a). 
Fig. 5. FC analysis differences at baseline + $\geq 48$ months. Activation maps are graphical representations of online suppl. Table $5 \mathrm{a}, \mathrm{b}$, where hot colors represent greater mean regional activation (i.e., between clusters for the local correlation analysis and between specific region and independent component for between-network FC analysis) in sMCI than in pMCI patients and cold colors represent lower activation group differences (i.e., cold colors reflect the opposite effect, greater mean regional activation in pMCI than in sMCI patients). Subcortical activation is represented in red. Activation values based on $T$ values (i.e., activation color bar range -5 to 5 ). FC, functional connectivity.
Local correlation differences at +48 months

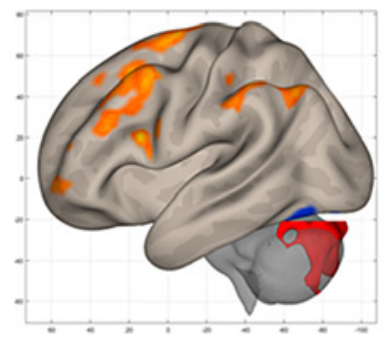

Left view

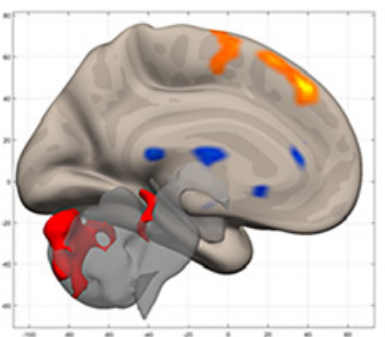

Left medial view

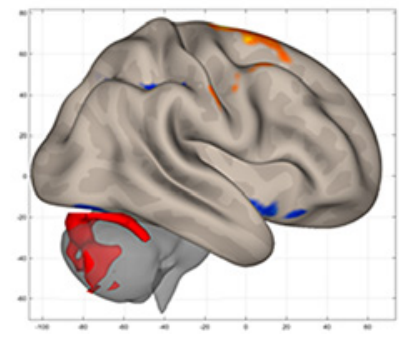

Right view

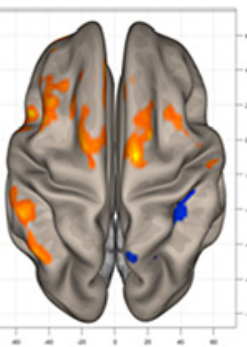

Superior view

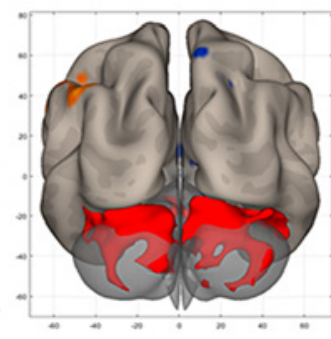

Posterior view
Between-network functional connectivity differences at +48 months

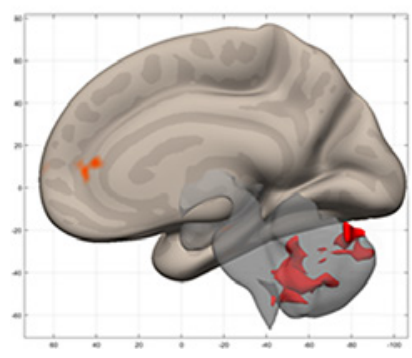

Right medial view

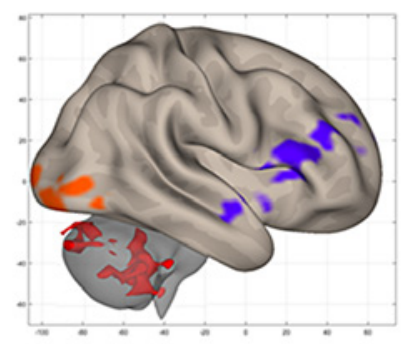

Right view

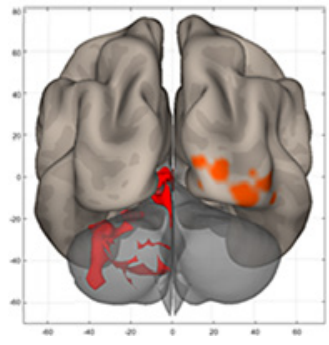

Posterior view
At 12-month follow-up, sMCI participants had greater local correlation than pMCI patients in the cerebellar network $(T=4.44$, $\mathrm{p}-\mathrm{FDR} \leq 0.001 ; T=4.13$, $\mathrm{p}-\mathrm{FDR} \leq 0.001)$, the DMN $(T=6.44$, p-FDR $\leq 0.001)$, and the $\mathrm{SN}(T=5.92$, $\mathrm{p}-\mathrm{FDR} \leq 0.001)$. Conversely, at 12 months pMCI patients had greater local correlation than sMCI patients in the visual network $(T=5.22$, $\mathrm{p}-\mathrm{FDR} \leq 0.001 ; T=4.30$, $\mathrm{p}-\mathrm{FDR}$ $\leq 0.001)$, the sensorimotor network $(T=6.08$, p-FDR $\leq 0.001 ; T=5.34, \mathrm{p}-\mathrm{FDR} \leq 0.001)$, and the $\mathrm{SN}(T=4.18$, $\mathrm{p}-\mathrm{FDR} \leq 0.001$; online suppl. Table $3 \mathrm{a}$ ). 
At 24 months, sMCI patients had greater local correlation than pMCI patients in the cerebellar network $(T=$ $5.22, \mathrm{p}-\mathrm{FDR} \leq 0.001)$ and sensorimotor network $(T=4.99$, $\mathrm{p}-\mathrm{FDR} \leq 0.001)$. Conversely, in pMCI participants, greater local correlation was observed in the $\mathrm{DMN}(T=6.03$, $\mathrm{p}-\mathrm{FDR} \leq 0.001 ; T=5.11, \mathrm{p}-\mathrm{FDR} \leq 0.001 ; T=4.88, \mathrm{p}$-FDR $\leq 0.001 ; T=4.21, \mathrm{p}-\mathrm{FDR} \leq 0.001)$, the sensorimotor network $(T=5.68, \mathrm{p}-\mathrm{FDR} \leq 0.001 ; T=4.97$, $\mathrm{p}-\mathrm{FDR} \leq 0.001)$, and the SN ( $T=4.46$, p-FDR $\leq 0.001)$ than in sMCI patients (online suppl. Table $4 \mathrm{a}$ ).

After 48 months, sMCI patients had greater local correlation than $\mathrm{pMCI}$ patients in the sensorimotor network $(T=10.32, \mathrm{p}-\mathrm{FDR} \leq 0.001 ; T=4.68, \mathrm{p}-\mathrm{FDR} \leq 0.001 ; T=$ $4.16, \mathrm{p}-\mathrm{FDR} \leq 0.001)$. Conversely, pMCI patients had greater local correlation than sMCI patients in the cerebellar network $(T=6.15, \mathrm{p}$-FDR $\leq 0.001 ; T=4.70$, p-FDR $\leq 0.001)$ and the sensorimotor network $(T=5.99$, p-FDR $\leq 0.001 ; T=4.35$, p-FDR $\leq 0.001$; online suppl. Table $5 \mathrm{a}$ ).

\section{Between-Network FC (Group-ICA)}

At baseline, sMCI patients had greater between-network FC than pMCI patients between the SN and frontoparietal network $(T=3.29$, p-FDR $=0.006)$, between the DMN and cerebellar network $(T=2.97, \mathrm{p}-\mathrm{FDR}=0.016$; $T=2.92$, p-FDR $=0.0018$ ), and between the DMN and $\mathrm{SN}$ $(T=2.74, \mathrm{p}-\mathrm{FDR}=0.008)$. Meanwhile, pMCI patients had greater between-network FC than sMCI patients between the $\mathrm{SN}$ and cerebellar network $(T=3.64, \mathrm{p}-\mathrm{FDR}=$ $0.002 ; T=2.13$, p-FDR $=0.048$; here, the multiple $T$-values correspond to multiple clusters within the identified component), between the DMN and $\mathrm{SN}(T=2.65$, p-FDR $=0.039 ; T=2.32$, p-DFR $=0.046)$, and between the DMN and cerebellar network $(T=2.22, \mathrm{p}-\mathrm{FDR}=0.048$; online suppl. Table $2 \mathrm{~b}$ ).

At the 12-month follow-up, greater between-network FC was observed in sMCI than in pMCI patients between the DMN and dorsal attention network $(T=4.05$, p-FDR $=0.032)$, the dorsal attention and cerebellar networks ( $T$ $=3.69, \mathrm{p}-\mathrm{FDR}=0.04)$, and the DMN and visual network $(T=3.6, \mathrm{p}-\mathrm{FDR}=0.025)$. Vice versa, greater betweennetwork FC was observed in pMCI than in sMCI patients between the DMN and cerebellar network $(T=4.24$, p$\mathrm{FDR}=0.016 ; T=3.93, \mathrm{p}-\mathrm{FDR}=0.049), \mathrm{DMN}$ and $\mathrm{SN}(T$ $=3.98, \mathrm{p}-\mathrm{FDR}=0.041), \mathrm{DMN}$ and sensorimotor network $(T=3.39$, p-FDR $=0.037)$, visual and sensorimotor networks $(T=4.09$, p-FDR $=0.014)$, and the visual network and SN $(T=4.01, \mathrm{p}-\mathrm{FDR}=0.037$; online suppl. Table $3 \mathrm{~b})$.

At 24 months, sMCI patients had greater between-network FC than pMCI patients between the DMN and SN $(T=3.24, \mathrm{p}-\mathrm{FDR}=0.009)$ and between the $\mathrm{DMN}$ and cerebellar networks $(T=2.99, \mathrm{p}-\mathrm{FDR}=0.006)$. Furthermore, pMCI patients had greater between-network FC than sMCI patients between the DMN and SN $(T=2.01$, $\mathrm{p}$-FDR $=0.049)$ and between the language and cerebellar networks $(T=2.71$, p-FDR $=0.037$; online suppl. Table $4 \mathrm{~b})$.

After 48 months, sMCI patients had greater betweennetwork FC than pMCI patients between the sensorimotor and cerebellar networks $(T=3.08, \mathrm{p}-\mathrm{FDR}=0.018)$ and between the dorsal attention and salience networks ( $T=$ $2.85, \mathrm{p}-\mathrm{FDR}=0.024)$. Meanwhile, pMCI patients had greater between-network FC than sMCI patients between the frontoparietal and salience networks $(T=3.9$, p-FDR $=0.003)$, language and salience networks $(T=2.64, \mathrm{p}$ $\mathrm{FDR}=0.047$ ), and between the frontoparietal and cerebellar networks ( $T=2.56$, p-FDR $=0.028$; online suppl. Table 5b).

\section{Discussion}

This work assessed functional brain connectivity at baseline, 12 months, 24 months, and $>48$ months (i.e., long-term) of pMCI and sMCI to further understand the differences associated with progression from aMCI to AD. To the best of our knowledge, this is the first study that compares stable MCI participants to MCI patients who progress to $\mathrm{AD}$ as observed over an extended period (i.e., >48 months). Stable MCI patients and MCI patients progressing to $\mathrm{AD}$ (pMCI) have, as known but in this population also observed, different cognitive profiles and, as shown here, different FC profiles. The disconnection syndrome hypothesis, which proposes a coordinated activity disruption among different brain regions, could explain these FC differences over time [30, 31]. It should be noted that the results reported here assess rs-fMRI data, which provide insight into the size of the effect of between-group differences in FC but not into their relationship with task-based performance. To determine whether the observed changes are related to compensation (i.e., brain reorganization with positive effects on task performance) or dedifferentiation (i.e., brain organization with no or negative effects on task performance), task-based fMRI studies would be necessary [32]. In our study, the voxel-to-voxel measures (i.e., local correlation and ICA) of rs-fMRI data compare regional activation differences between groups without a priori knowledge and thus provide data-driven insights into brain network dynamics during the resting state. 
Patients with aMCI are not cognitively the same, and pMCI and sMCI patients had different cognitive profiles. Patients who progressed to AD consistently had lower global cognition since pMCI patients had higher CDR-SB at all follow-up time-points; lower MMSE and MoCA at the 12-, 24-, and +48-month follow-ups; and higher ADAS-Cog at the 12-month follow-up than the baseline psychometric evaluation. Regarding within-group changes over time, pMCI patients had markedly different cognitive trajectories compared to sMCI patients. While sMCI patients only had differences between the baseline and long-term MMSE evaluations, the pMCI patients had higher CDR-SB and lower MMSE scores at all follow-up time-points, higher ADAS-Cog at the year follow-up, and lower MoCA scores at the 24- and +48-month follow-ups than the baseline evaluation. As time progressed, the patient cognition deteriorated more in the pMCI group than the baseline evaluation as expected. In this study, aMCI patient progression to AD was consistently associated with a deterioration in global cognition over time.

Over the different time-points, the local correlation appears to be affected in the sensorimotor network throughout the observation period, while in the $\mathrm{SN}$ and DMN it appears to be affected during the early observational points (i.e., baseline and at 12 months) only. The predictive value of these findings should be further investigated. In regard to between-network FC, the SN and DMN (i.e., each network had 4 ICs with FC network activation differences) had more ICs with statistically significant between-group differences in FC at baseline, the DMN (i.e., 5 independent components with activation differences) at 12 months, the DMN and SN (i.e., 3 independent components with activation differences) at 24 months, and the $\mathrm{SN}$ (i.e., 4 independent components with activation differences) at $>48$ months. The DMN and SN thus repeatedly showed between-network FC differences between sMCI and pMCI groups across the different observational points and seem to be most affected.

Reduced regional resting-state activity in aMCI patients compared to healthy participants has been found in the posterior cingulate cortex, right angular gyrus, right parahippocampal gyrus, left fusiform gyrus, left supramarginal gyrus, and bilateral middle temporal gyri [33]. It has been suggested that the topological architecture of the functional connectome in aMCI patients is disrupted and that its integrity is correlated to the patient's memory performance [15]. The cascading network failure model could serve as an explanation of why the DMN and SN are most affected when comparing sMCI and pMCI patients. The cascading network failure model alludes to the disconnection effect proliferating to structurally and functionally connected regions, eventually leading to failure of the entire system [34]. In $\mathrm{AD}$, the cascading network failure begins in the posterior DMN, causing a connectivity overload (i.e., a process "analogous to cascading failures seen in power grids triggered by local overloads proliferating to downstream nodes eventually leading to widespread power outages, or systems failures"; Jones et al. [34], p. 547) that precedes a structural and functional decline; in consequence, a transient load-shifting compensatory process is triggered. The shifting of processing burden produces a compensatory sequential increase in connectivity, which propagates in a posterior-ventral to an anterior-dorsal gradient, mirroring the spatiotemporal patterns of histopathological disease progression in $\mathrm{AD}$ [34]. Previous cross-sectional studies with neuroimage sampling at different time-points that assessed aMCI patients over a 2 -year and a 3 -year window reported more disruptions and connectivity changes in pMCI than in sMCI patients compared to HC $[17,19-21]$. These changes ranged from whole-brain connectivity changes to local FC disruptions $[17,19]$. Considering that the histopathological disease progression has a direct effect on the neuronal network distribution in $\mathrm{AD}$, aMCI and specifically pMCI patients should be associated with topological network architecture with intermediate characteristics between healthy and AD participants. The entorhinal and hippocampal regions, integral parts of the DMN and key structures affected in the onset of $\mathrm{AD}$, have been found to have reduced between-network FC in pMCI patients compared to HC [20]. Additionally, a gradual and progressive decrease in seed-to-voxel between-network FC in other regions of the DMN was observed in pMCI compared to sMCI patients [21].

\section{Limitations and Future Perspectives}

Common problems in cross-sectional fMRI studies that obtain neuroimages at different time-points are participant dropout and head motion. This study is no exception as only 79 participants had adequate baseline scans, 78 participants had valid 1-year follow-up scans, 51 participants had valid 24-month follow-up scans, and 22 participants had a valid long-term follow-up scan. As the number of participants dwindles, findings become less reliable, thus limiting the number of valid between-group comparisons and the interpretability of these results. Furthermore, more sMCI than pMCI patients were consistently included in the different FC analyses, which is consistent with the yearly progression to $\mathrm{AD}$ rate found in the literature (i.e., 10-34\%). Another limitation regarding the 
long-term interpretation of our findings concerns the variability in the time-point at which the long-term group of patients was assessed (i.e., aMCI: median, 61 months; range, 46-86 months; interquartile range, 48-73). Based on the wide time range for the long-term neuroimaging assessment, cautious interpretation of these results is warranted. The main goal of this study was to monitor disease progression at different observational points. A cross-sectional design study with 4 time-points allowed for between-group comparisons in a +4 -year window, which we considered to be a strength for an rs-fMRI paradigm. A longitudinal design assessing within-subject changes throughout time was considered; however, this limited the cohort size with 4 cross-sectional time-points to 22 subjects, thus reducing the statistical power of the study. In addition, we considered that the advantage of performing an rs-fMRI study was the ability to detect between-group differences more adequately than withinsubject changes. To assess longitudinal within-subject changes, we considered that other neuroimaging techniques such as EEG may be more suitable than fMRI studies. The aim of this study was to study the between-group differences between aMCI patients who do and do not progress to $\mathrm{AD}$. ADNI has primarily included and followed up overtime aMCI patients. We considered comparing aMCI patients to cognitively unimpaired participants; however, only 33 healthy controls had a baseline and a 12-month follow-up fMRI, while 7 had a 24 -month follow-up, and none a long-term BOLD contrast sequence available. Previous ADNI studies have performed between-group analysis between healthy controls and aMCI patients (i.e., including comparisons to both sMCI and $\mathrm{pMCI}$ ) and between $\mathrm{pMCI}$ and $\mathrm{AD}$ patients crosssectionally at baseline and after a 12-month follow-up; hence, we decided not to perform such analyses. Finally, we also report that 7 aMCI patients had cognitive reversion (rMCI). Cognitive reversion is an interesting phenomenon; however, FC analysis using the current sample was not performed due to the small sample size. Future work is needed to assess FC differences between rMCI and both sMCI and pMCI patients, as well as differences between sMCI and pMCI at $\geq 48$ months with larger sample sizes.

\section{Conclusion}

sMCI patients and MCI patients progressing to $\mathrm{AD}$ exhibit differences in cognitive trajectories, as well as in brain FC. As expected, progression to AD in aMCI patients was consistently associated with a reduced global cognition over time. In this study, we found that more between-network FC than local correlation differences were present between sMCI and pMCI at different observational points across a period of over 4 years. The DMN and SN repeatedly showed both local correlation and between-network FC differences between the sMCI and pMCI patient groups. Brain network dynamics at rest are different between patients with MCI who progress to dementia and MCI patients who remain cognitively stable; however, more research is necessary to understand the processes that might be involved with FC compensation and loss of network integrity.

\section{Statement of Ethics}

We certify that no fraudulent or manipulated data were used, that all reported data and results are authentic and valid, that no content of the submission has been plagiarized, and that the work has not been published before nor is being considered for publication in another journal. This study was approved by the Institutional Review Board of each participating hospital. Abiding by the Declaration of Helsinki, patient anonymity was guaranteed.

\section{Conflict of Interest Statement}

This research did not receive any specific grant from funding agencies in the commercial or not-for-profit sectors, and the authors report no conflict of interest.

\section{Funding Sources}

This study was supported by CONACyT (Consejo Nacional de Ciencia y Tecnología) Grant \#440591 (grant recipient J.D.M., career grant).

\section{Author Contributions}

The author listing is complete, and all authors have contributed significantly to the work. Original idea, data acquisition, data analysis, manuscript writing, and manuscript correction were by J.D.M.U. Data extraction, data analysis, and manuscript writing were carried out by R.S.M. Original idea, data analysis, and manuscript correction were done by P.P.D.D. Original idea, data analysis, manuscript writing, and manuscript correction were done by N.M.M.

\section{Data Availability Statement}

The data that support the findings of this study can be available upon request. Raw data are available at http://adni.loni.usc.edu/ data-samples/access-data/. 


\section{References}

1 Petersen RC, Doody R, Kurz A, Mohs RC, Morris JC, Rabins PV, et al. Current concepts in mild cognitive impairment. Arch Neurol. 2001 Dec;58(12):1985-92.

2 Sperling RA, Aisen PS, Beckett LA, Bennett DA, Craft S, Fagan AM, et al. Toward defining the preclinical stages of Alzheimer's disease: recommendations from the National Institute on Aging-Alzheimer's Association workgroups on diagnostic guidelines for Alzheimer's disease. Alzheimers Dement. 2011 May; 7(3):280-92.

3 Rozzini L, Chilovi BV, Conti M, Bertoletti E, Delrio I, Trabucchi M, et al. Conversion of amnestic Mild Cognitive Impairment to dementia of Alzheimer type is independent to memory deterioration. Int J Geriatr Psychiatry. 2007 Dec;22(12):1217-22.

4 Schmidtke K, Hermeneit S. High rate of conversion to Alzheimer's disease in a cohort of amnestic MCI patients. Int Psychogeriatr. 2008 Feb;20(1):96-108.

5 Ottoy J, Niemantsverdriet E, Verhaeghe J, De Roeck E, Struyfs H, Somers C, et al. Association of short-term cognitive decline and MCIto-AD dementia conversion with CSF, MRI, amyloid- and 18F-FDG-PET imaging. Neuroimage Clin. 2019;22:101771.

6 Stephan KE, Friston KJ. Functional connectivity. In: Squire LR, editor. Encyclopedia of neuroscience. Cambridge, Massachusetts: Academic Press; 2009. p. 391-7. Available from: http://dx.doi.org/10.1016/b978008045046-9.00308-9.

7 Lv H, Wang Z, Tong E, Williams LM, Zaharchuk $\mathrm{G}$, Zeineh $\mathrm{M}$, et al. Resting-state functional MRI: everything that nonexperts have always wanted to know. AJNR Am J Neuroradiol. 2018 Aug;39(8):1390-9.

8 Deshpande G, LaConte S, Peltier S, Hu X. Integrated local correlation: a new measure of local coherence in fMRI data. Hum Brain Mapp. 2009 Jan;30(1):13-23.

9 Wylie KP, Kronberg E, Maharajh K, Smucny J, Cornier MA, Tregellas JR. Between-network connectivity occurs in brain regions lacking layer IV input. Neuroimage. 2015 Aug 1;116:50-8.

10 Grady CL, Protzner AB, Kovacevic N, Strother SC, Afshin-Pour B, Wojtowicz M, et al. A multivariate analysis of age-related differences in default mode and task-positive networks across multiple cognitive domains. Cereb Cortex. 2010 Jun;20(6):1432-47.

11 Sambataro F, Murty VP, Callicott JH, Tan HY, Das S, Weinberger DR, et al. Age-related alterations in default mode network: impact on working memory performance. Neurobiol Aging. 2010 May;31(5):839-52.
12 Geerligs L, Renken RJ, Saliasi E, Maurits NM, Lorist MM. A brain-wide study of age-related changes in functional connectivity. Cereb Cortex. 2015 Jul;25(7):1987-99.

13 Sullivan MD, Anderson JAE, Turner GR, Spreng RN. Alzheimer's Disease Neuroimaging Initiative. Intrinsic neurocognitive network connectivity differences between normal aging and mild cognitive impairment are associated with cognitive status and age. Neurobiol Aging. 2019 Jan;73:219-28.

14 Wang J, Zuo X, Dai Z, Xia M, Zhao Z, Zhao $\mathrm{X}$, et al. Disrupted functional brain connectome in individuals at risk for Alzheimer's disease. Biol Psychiatry. 2013 Mar 1;73(5): 472-81.

15 Wang L, Li H, Liang Y, Zhang J, Li X, Shu N, et al. Amnestic mild cognitive impairment: topological reorganization of the defaultmode network. Radiology. 2013 Aug;268(2): 501-14.

16 Franzmeier N, Caballero MÁA, Taylor ANW, Simon-Vermot L, Buerger K, Ertl-Wagner B, et al. Resting-state global functional connectivity as a biomarker of cognitive reserve in mild cognitive impairment. Brain Imaging Behav. 2017 Apr;11(2):368-82.

17 Pereira JB, Mijalkov M, Kakaei E, Mecocci P, Vellas B, Tsolaki M, et al. Disrupted network topology in patients with stable and progressive mild cognitive impairment and Alzheimer's disease. Cereb Cortex. 2016 Aug;26(8): 3476-93.

18 Filippi M, Basaia S, Canu E, Imperiale F, Magnani G, Falautano $\mathrm{M}$, et al. Changes in functional and structural brain connectome along the Alzheimer's disease continuum. Mol Psychiatry. 2020 Jan;25(1):230-9.

19 Sun Y, Bi Q, Wang X, Hu X, Li H, Li X, et al. Prediction of conversion from amnestic mild cognitive impairment to Alzheimer's disease based on the brain structural connectome. Front Neurol. 2019 Jan 10;9:1178.

20 Delli Pizzi S, Punzi M, Sensi SL. Alzheimer's Disease Neuroimaging Initiative. Functional signature of conversion of patients with mild cognitive impairment. Neurobiol Aging. 2019 Feb;74:21-37.

21 Li Y, Wang X, Li Y, Sun Y, Sheng C, Li H, et al. Abnormal resting-state functional connectivity strength in mild cognitive impairment and its conversion to Alzheimer's disease. Neural Plast. 2016;2016. 4680972.

22 Mueller SG, Weiner MW, Thal LJ, Petersen RC, Jack CR, Jagust W, et al. Ways toward an early diagnosis in Alzheimer's disease: the Alzheimer's Disease Neuroimaging Initiative (ADNI). Alzheimers Dement. 2005 Jul;1(1): 55-66.
23 Shaw LM, Vanderstichele H, Knapik-Czajka M, Figurski M, Coart E, Blennow K, et al. Qualification of the analytical and clinical performance of CSF biomarker analyses in ADNI. Acta Neuropathol. 2011 May;121(5): 597-609.

24 Alzheimer's Disease Neuroimaging Initiative. ADNI General Procedures Manual 2010. Available from: http://adni.loni.usc.edu/ methods/documents/. Accessed 2018 Nov 15

25 McKhann GM, Knopman DS, Chertkow H, Hyman BT, Jack CR Jr, Kawas CH, et al. The diagnosis of dementia due to Alzheimer's disease: recommendations from the National Institute on Aging-Alzheimer's Association workgroups on diagnostic guidelines for Alzheimer's disease. Alzheimers Dement. 2011 May;7(3):263-9.

26 O'Bryant SE, Waring SC, Cullum CM, Hall J, Lacritz L, Massman PJ, et al. Staging dementia using Clinical Dementia Rating Scale Sum of Boxes scores: a Texas Alzheimer's research consortium study. Arch Neurol. 2008 Aug; 65(8):1091-5.

27 Whitfield-Gabrieli S, Nieto-Castanon A Conn: a functional connectivity toolbox for correlated and anticorrelated brain networks. Brain Connect. 2012;2(3):125-41.

28 Ashburner J. A fast diffeomorphic image registration algorithm. Neuroimage. 2007 Oct 15;38(1):95-113.

29 Calhoun VD, Adali T, Pearlson GD, Pekar JJ. A method for making group inferences from functional MRI data using independent component analysis. Hum Brain Mapp. 2001 Nov; 14(3):140-51.

30 Delbeuck X, Collette F, Van der Linden M. Is Alzheimer's disease a disconnection syndrome? Evidence from a crossmodal audiovisual illusory experiment. Neuropsychologia. 2007 Nov 5;45(14):3315-23.

31 Vallet GT, Hudon C, Simard M, Versace R. The disconnection syndrome in the Alzheimer's disease: the cross-modal priming example. Cortex. 2013 Oct;49(9):2402-15.

32 Cabeza R, Anderson ND, Locantore JK, McIntosh AR. Aging gracefully: compensatory brain activity in high-performing older adults. Neuroimage. 2002 Nov;17(3):1394402.

33 Lau WK, Leung MK, Lee TM, Law AC. Resting-state abnormalities in amnestic mild cognitive impairment: a meta-analysis. Transl Psychiatry. 2016 Apr 26;6(4):e790.

34 Jones DT, Knopman DS, Gunter JL, GraffRadford J, Vemuri P, Boeve BF, et al. Cascading network failure across the Alzheimer's disease spectrum. Brain. 2016 Feb;139(Pt 2): 547-62.
Functional Connectivity and AD Progression
Dement Geriatr Cogn Disord Extra 2021;11:235-249 DOI: $10.1159 / 000518233$ 